\title{
Electron Properties and Thermal Decomposition Behaviors for HMX/HTPB Plastic Bonded Explosives
}

\author{
Zheng-Hua He ${ }^{a}$, Yao-Yao Huang ${ }^{a}$, Guang-Fu Ji ${ }^{a}$, Jun Chen ${ }^{b}$, Qiang Wu ${ }^{*}, a$
}

${ }^{a}$ National Key Laboratory of Shock Wave and Detonation Physics, Institute of Fluid Physics, China Academy of Engineering Physics, Mianyang 621900, Sichuan, China.

${ }^{b}$ National Key Laboratory of Computational Physics, Institute of Applied Physics and Computational Mathematics, Beijing 100088, China.

Corresponding author:

Qiang $\mathrm{Wu}$

Email: wuqianglsd@163.com

Telephone: 0086-0816-2491024 


\section{Supporting Information}

Model construction (see Fig. S1): The initial HTPB molecules derive from the homo-polymerizing of three or four butadiene units, terminated with hydroxyl. Four HTPB molecules consist of an amorphous 3-dimension (3D) structure (a and b axis are equal to that of the corresponding crystal surface, while c axis is determined according to the density of HTPB), which further undergoes geometry optimization to obtain more reasonable configuration. After that, the HTPB molecules are drawn out from the 3D structure to obtain the molecule slab. The slab is adsorbed on HMX surface by annealing simulation, and another geometry optimization is carried out to get stable adsorption structure. All the procedures above are carried out based on the software package of Materials Studio, and the classical COMPASS force field is employed for the model construction and optimization. Finally, the adsorption structure is further optimized using more precise method of DFTB, and the surplus vacuum is cut according to interaction distance of HMX and HTPB to construct the 3D periodic model.

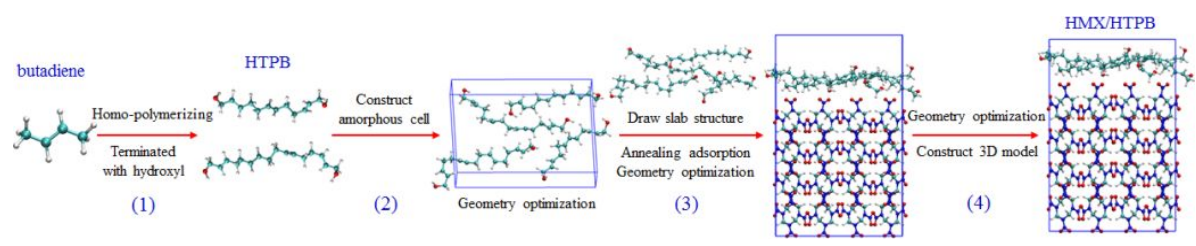

Fig. S1 the sketch for construction of HMX/HTPB composite model.

Formula S1: Here, $\rho_{0}, \rho_{1}$, and $\rho_{2}$, denote the density of HMX/HTPB composite, HMX and HTPB; $m_{1}$ and $m_{2}$ represent the mass of HMX and HTPB; $V_{1}$ and $V_{2}$ represent volume of HMX and HTPB; $\alpha$ represents mass ratio of HTPB to HMX.

$$
\rho_{0}=\frac{m_{1}+m_{2}}{V_{1}+V_{2}}=\frac{1+\alpha}{\frac{1}{\rho_{1}}+\frac{\alpha}{\rho_{2}}} \quad\left(m_{2} / m_{1}=\alpha\right)
$$

Table S1 $R_{\mathrm{c}}$ for different chemical bonds at different reaction stages

\begin{tabular}{ccccccccccc}
\hline Bonds & C-C & C-N & C-O & C-H & N-N & N-O & N-H & O-O & O-H & H-H \\
\hline$R_{\mathrm{c}} / \AA$ & 1.77 & 1.74 & 1.74 & 1.31 & 1.70 & 1.70 & 1.28 & 1.70 & 1.28 & 0.85 \\
\hline
\end{tabular}




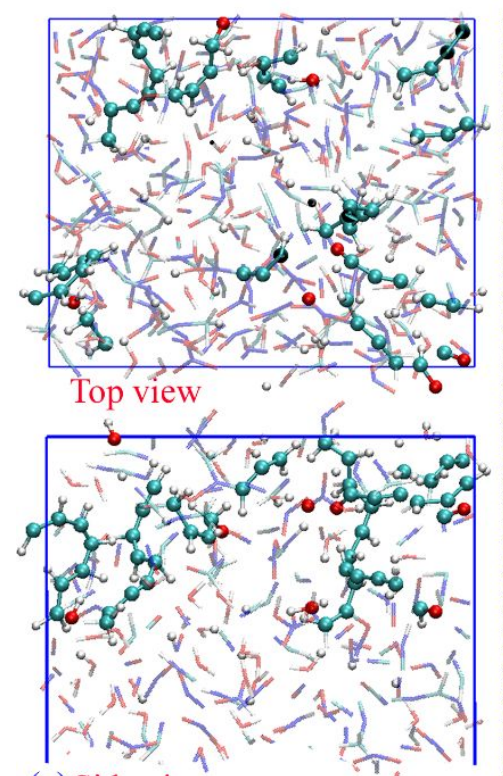

(a) Side view

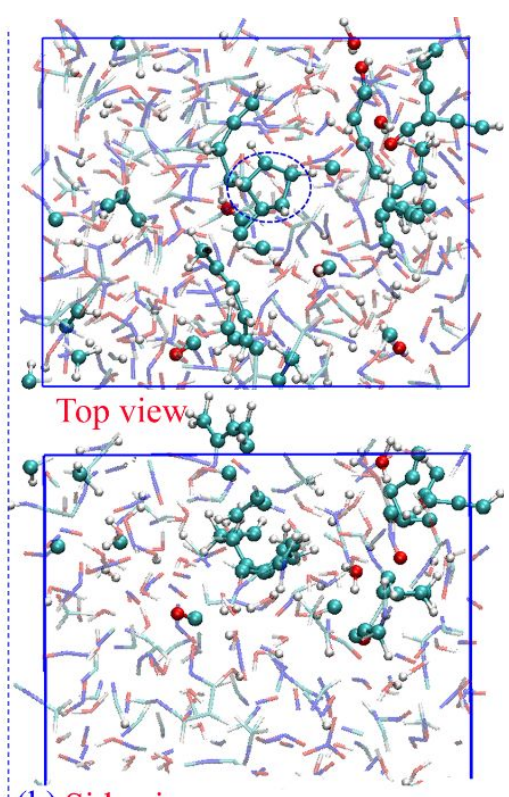

(b) Side view

Fig. S2 Snapshot of the HTPB molecular chains within HMX(100)/HTPB composites at 3 ps: (a) initial configuration, (b) compressed configuration.

Difference of the reaction properties. Comparing the reaction process of these different HMX/HTPB composites (see Fig. 7 and Fig. S3-S4), the primary products and their concentration evolution trends are similar with each other. Only a little differences about their specific concentration are observed. As for initial configurations, the HMX(010)/HTPB system displays a slight lower reaction activity than the others. The concentrations of $\mathrm{H}_{2} \mathrm{O}$ and $\mathrm{NO}$ are lower than that of the other two conditions, but the density of $\mathrm{N}_{2} \mathrm{O}$ is larger than the others. $\mathrm{HMX}(001) / \mathrm{HTPB}$ system is more beneficial for formation of NO. As for the compressed configuration, the differences among different models become more trivial, illustrating that the thermal response does not possess significant anisotropy.
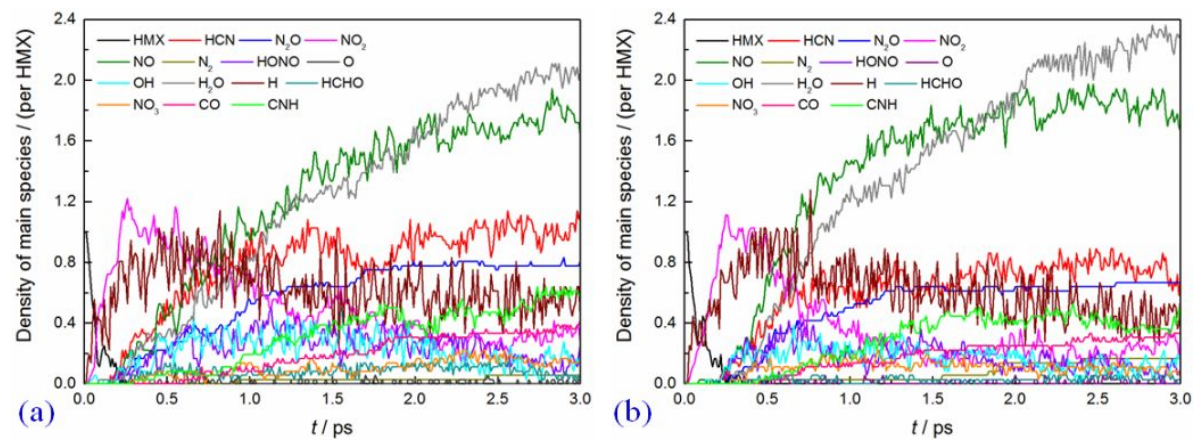

Fig. S3 Concentration evolutions of main species involved in HMX(010)/HTPB thermal decomposition: (a) initial configuration, (b) compressed configuration. 

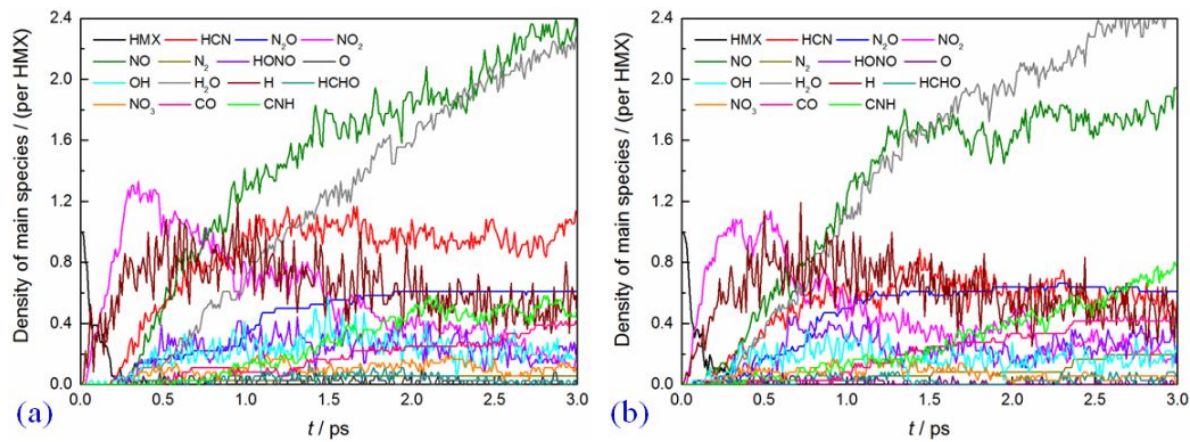

Fig. S4 Concentration evolutions of main species involved in HMX(001)/HTPB thermal decomposition: (a) initial configuration, (b) compressed configuration.
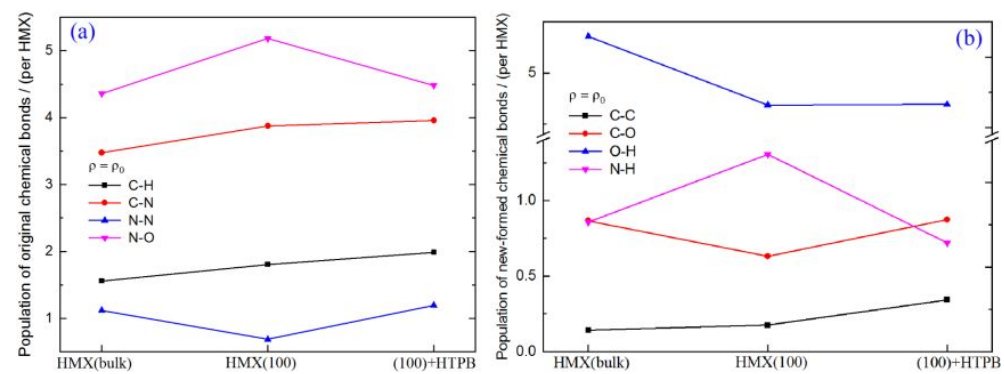

Fig. S5 Final population of main chemical bonds: (a) original chemical bonds, (b) new formed chemical bonds. 